\title{
Surface properties probed by second-harmonic and sum-frequency generation
}

\section{Y. R. Shen}

Department of Physics, University of California, Materials and Chemical Sciences Division, Lawrence Berkeley Laboratory, Berkeley, California 94720 , USA

Optical second-harmonic generation and the related technique of infrared-visible light sum-frequency generation are extremely versatile tools for studies of many kinds of surfaces and interfaces. With the help of ultra-short laser pulses, they can be used to monitor surface dynamics and reactions with sub-picosecond time resolution.

OPTICAL second-order harmonic generation is an exciting new technique for studying surfaces and interfaces. Surface science plays an important role in disciplines ranging from physics and chemistry to biology and electronics, where reliable characterization of surfaces and interfaces is essential. Accordingly, many tools for surface analysis have been developed over the years but most are limited in their application ${ }^{1}$. For example, electron scattering and diffraction, photoemission, Auger spectroscopy and mass spectroscopy can be operated only in a vacuum. Optical techniques can generally be applied to any interface accessible to light but they may not have enough surface specificity and sensitivity and can therefore be used only if the signal contributed by the bulk can be suppressed ${ }^{2}$.

Recently, several laser techniques have been developed for surface probing ${ }^{3}$. Among these, second-harmonic generation (SHG) has received much attention because of its simplicity, surface specificity and versatility.

The SHG technique is sufficiently sensitive to detect less than a monolayer of molecules adsorbed on a surface. As a surface probe, it has several attractive features. Being a laser-excited coherent optical process, surface SHG is highly directional. It is therefore suitable for non-detrimental, in situ remote-sensing studies and can be used for monitoring surfaces in a real environment. Sub-picosecond laser pulses can be used for probing, giving the technique the potential for sub-picosecond time resolution and making it useful for in situ real-time probing of fast surface dynamics and reactions. Because laser excitation is capable of high spatial and spectral resolutions, it also allows in situ mapping of the molecular arrangement and composition of a surface monolayer. These advantages, together with its general applicability to all types of interfaces, make SHG a unique and versatile tool for surface studies.

The SHG method is based on the principle that a second-order nonlinear optical process is forbidden in a medium with inversion symmetry, but such symmetry will necessarily be broken at an interface, where the nonlinear processes are therefore allowed. Consider an optical wave at frequency $\omega$ impinging on a medium. The wave can induce a dipole oscillation of each molecule in the medium. Because a molecule behaves like an anharmonic oscillator, overtone oscillations are excited at frequencies $2 \omega, 3 \omega$ and so on, as well as the fundamental at $\omega$. Oscillating dipoles emit electromagnetic radiation, and hence one expects output radiation from the medium at frequencies $\omega, 2 \omega, 3 \omega$ and so on. In the SHG technique, one focuses attention on the frequency $2 \omega$. For a driven anharmonic oscillator, the induced-dipole component, $p(2 \omega)$, (which is the source of the second-harmonic radiation) is proportional to the quantity $E(\omega) E(\omega)$, the 'self product' of the incoming field of strength $E$, at frequency $\omega$. The induced second-harmonic dipole per unit volume, $P^{(2)}(2 \omega)$, can be written as

$$
P^{(2)}(2 \omega)=\chi^{(2)} E(\omega) E(\omega)
$$

where $\chi^{(2)}$ is known as the nonlinear susceptibility and is a characteristic coefficient of the medium. If the medium has

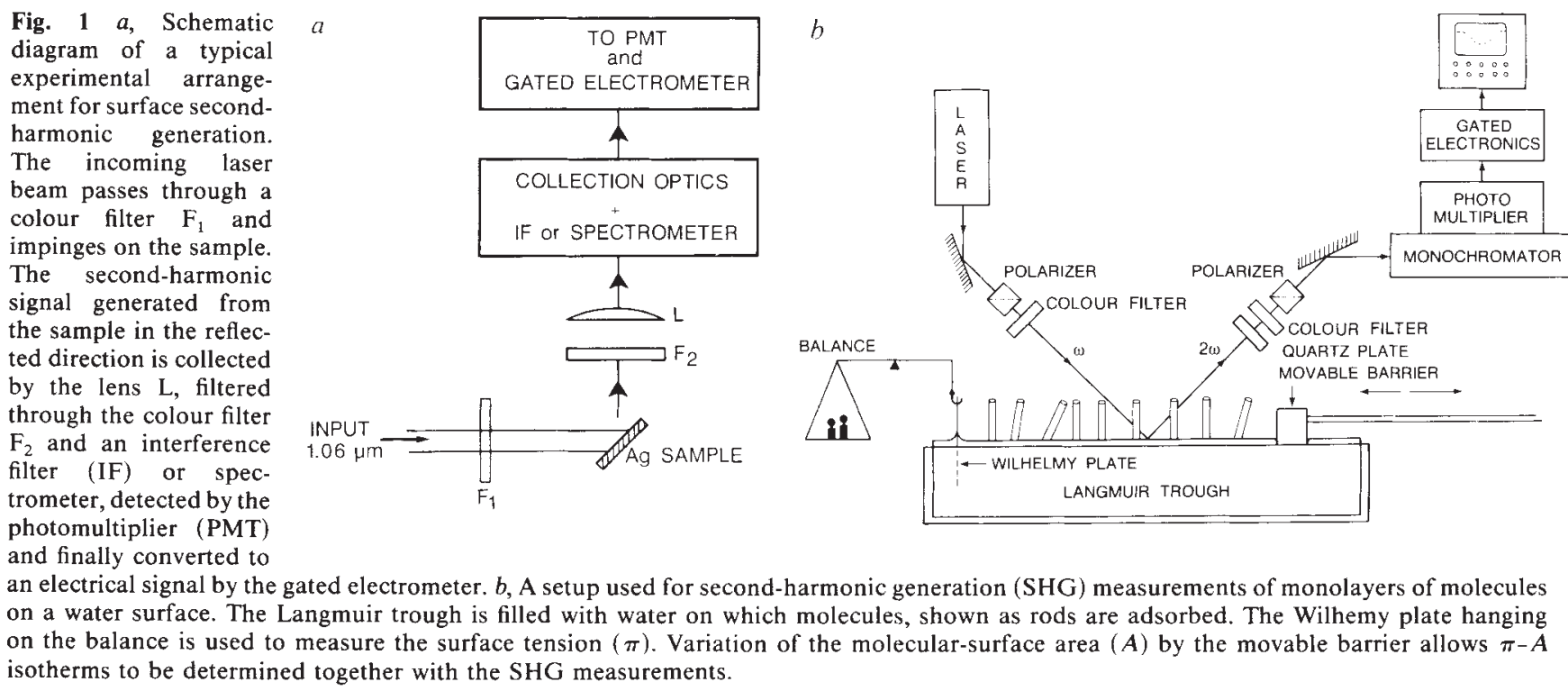

Fig. 1 a, Schematic ment for surface secondthe sample in the reflected direction is collected by the lens $\mathrm{L}$, filtered through the colour filter $\mathrm{F}_{2}$ and an interference filter (IF) or spectrometer, detected by the hotomultiplier (PMT) on a water surface. The Langmuir trough is filled with water on which molecules, shown as rods are adsorbed. The Wilhemy plate hanging isotherms to be determined together with the SHG measurements. 
inversion symmetry, the incoming fields $E(\omega)$ and $-E(\omega)$ must induce in the medium dipoles of $P(2 \omega)$ and $-P(2 \omega)$ respectively. This, however, is not consistent with equation (1) unless $\chi^{(2)}=0$, indicating that SHG is forbidden. At an interface, the inversion symmetry must be broken, and radiation at frequency $2 \omega$ is therefore no longer forbidden. Consequently, SHG is highly surface specific.

\section{Theory}

The sub-monolayer sensitivity of SHG was noticed in $1973^{10}$ but received little attention. It was rediscovered in a search for a better understanding of the phenomenon of surface-enhanced Raman scattering ${ }^{11,12}$. Several researchers suggested that this scattering arises because of enhancement of the local field at points on a rough metal surface ${ }^{13,14}$ Raman scattering can be regarded as a two-photon nonlinear optical process, in which one photon is absorbed and another emitted. It was therefore suggested that other nonlinear optical processes should be similarly enhanced on rough metal surfaces ${ }^{15}$. Indeed, with a $1.06-\mu \mathrm{m}$ laser pump, SHG is enhanced by a factor of $\sim 10^{4}$ on a roughened silver surface ${ }^{15}$. The signal is so strong that submonolayers of adsorbates on roughened silver can be monitored with a continuous-wave (CW) diode $l a s e r^{16}$. An approximate estimate of the signal strength indicates that even without surface enhancement, it should be possible to detect SHG from a molecular monolayer.

The theory of surface SHG has been well developed ${ }^{4-7}$, following the early work of Bloembergen and Pershan ${ }^{8}$, and we will discuss it only briefly. The physical origin of the surface nonlinearity arises from two sources ${ }^{6,7}$ : structural discontinuity and field discontinuity at the interface. Which is the more important depends on the structure of the interfacial system. Structural discontinuity clearly dominates at a semiconductor surface with dangling bonds, for example, or where a monolayer of polar molecules is adsorbed with a preferred orientation at an interface. Field discontinuity, on the other hand, may dominate on a liquid or glass surface, where the surface structure is not very different from that in the bulk. When higher-order effects are taken into account, the bulk should also contribute to the secondharmonic signal but it can be shown that the contribution is usually smaller than that from the surface. In any case, a surface modification should certainly be discernible by surface SHG even in the presence of a significant bulk contribution.

The strength of surface SHG can be estimated from equation A-2 (ref. 9; see panel below) typically, $\left|\chi_{s}^{(2)}\right|$ is of the order of $10^{-15}$ e.s.u. (here subscript s denotes a surface property). Assuming $L \approx 1, \varepsilon \approx 4$ and $\sec ^{2} \theta \approx 2$, for the parameters in equation A-2, a laser beam with intensity $I(\omega) \approx 10 \mathrm{MW} \mathrm{cm}^{-2}$, crosssectional area $A \approx 0.2 \mathrm{~cm}^{2}$ and pulse duration $T \approx 10 \mathrm{~ns}$ at a wavelength of $1.06 \mu \mathrm{m}$ should generate a second-harmonic output of $\approx 10^{3}$ photons per pulse. Signal strength is readily detectable with a photomultiplier, and points clearly to the sub-monolayer sensitivity of the technique. Equation A-2 indicates that the sensitivity should increase with laser intensity. Sensitivity is, however, eventually limited by the onset of surface damage at high beam intensity; to avoid surface damage or perturbation, the laser energy per unit area must be kept below a certain threshold value.

\section{Applications}

The experimental arrangement for surface SHG is fairly simple (Fig. 1). In general, pulsed lasers are used for excitation but $\mathrm{CW}$ lasers can also be used in some cases. The large difference in frequency between the fundamental $(\omega)$ and the second harmonic $(2 \omega)$ makes filtering of the signal against unwanted background straightforward. Because the output is highly directional, spatial filtering can be used effectively to block most of the fluorescence or stray light from the sample area.

In the following, we discuss applications of surface SHG to various disciplines in surface science and give examples to illustrate the potential of the technique.

Metal surfaces. One of the most flourishing areas of research in
Surface SHG is an electromagnetic phenomenon governed by Maxwell's equations. The source of the output radiation at frequency $2 \omega$ is a surface polarization

$$
\mathbf{P}_{s}^{(2)}(2 \omega)=\chi_{s}^{(2)}: \mathbf{E}(\omega) \mathbf{E}(\omega)
$$

induced by the incoming laser field $\mathbf{E}(\omega)$ in the interface layer, where the surface susceptibility tensor $\chi_{s}^{(2)}$ is a characteristic constant of the interface layer. This corresponds to a sheet of coherently driven dipoles oscillating at $2 \omega$. Like an array of antennal, these oscillating dipoles radiate coherently in a well defined direction. Solution of Maxwell's equations with $\mathbf{P}^{(2)}(2 \omega)$ as the source term yields a second-harmonic output $S(2 \omega)$ in the reflected direction (assuming an air/solid interface) given by

$$
\begin{aligned}
S(2 \omega)= & {\left[32 \pi^{3} \omega \sec ^{2} \theta / \hbar c^{3} \varepsilon(\omega)^{1 / 2}(2 \omega)\right] \mid \mathbf{L}(2 \omega) } \\
& \times \chi_{s}^{(2)}:\left.\mathbf{L}(\omega) \mathbf{L}(\omega)\right|^{2} I^{2}(\omega) A T \text { photons per pulse }
\end{aligned}
$$

where $\theta$ is the angle of incidence of the incoming laser beam, $\varepsilon(\omega)$ is the dielectric constant at $\omega, \mathbf{L}(\omega)$ is a quantity that takes into account the Fresnel factor, and the beam polarization at $\omega$, and $I(\omega), A$ and $T$ are the intensity, the beam cross-section at the interface and the pulsewidth of the laser beam, respectively.

In equations (A-1) and (A-2), $\chi_{s}^{(2)}$ is a third-rank tensor which, in Cartesian coordinates, is defined by a set of 27 tensor elements $\left(\chi_{s}^{(2)}\right)_{i j k}$, with $i, j$ and $k$ representing $x, y$ or $z$. For example, according to equation (A-1), the incoming field components $E_{j}$ and $E_{k}$ can induce a surface-polarization component

$$
\left(P_{s}^{(2)}(2 \omega)\right)_{i}=\left(\chi_{s}^{(2)}\right)_{i j k} E_{j}(\omega) E_{k}(\omega)
$$

which is responsible for generation of the $i$-component of the second-harmonic field. Dictated by the symmetry of the interfacial system, many elements of $\chi_{s}^{(2)}$ can be vanishing or dependent on the others. With different combinations of input and output beam polarizations, the independent non-vanishing elements of $\chi_{s}^{(2)}$ can

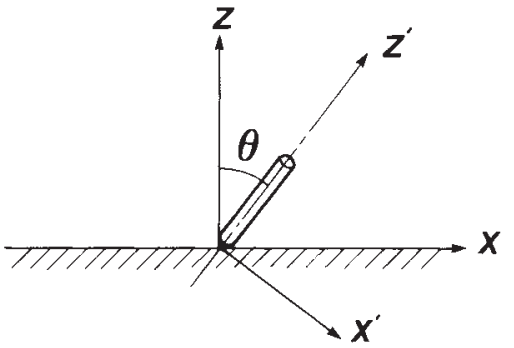

The orientation of a rod-like molecule at an interface. The angle $\theta$ between the molecular axis $z^{\prime}$ and the surface normal $z$ is measured by SHG using different combinations of input and output beam polarizations.

be deduced from the SHG measurements. In some cases, this also allows the average molecular orientation to be determined.

Consider the case where the molecule has a well defined long molecular axis and its nonlinear polarizability (or hyperpolarizability) tensor $\alpha^{(2)}$ is dominated by a single tensor element $\alpha_{z^{\prime} z^{\prime} z^{\prime}}^{(2)}$ with $\mathbf{z}^{\prime}$ along the molecular axis. One can easily relate $\alpha_{z^{\prime} z^{\prime} z^{\prime}}^{(2)}$ to the various elements of $\chi_{s}^{(2)}$ in the laboratory coordinates $(x, y, z)$ by a coordinate transformation. If $\theta$ is the angle between $z^{\prime}$ and the surface normal $\boldsymbol{z}$ (see figure) and the molecules have a random azimuthal distribution, then

$$
\begin{aligned}
\left(\chi_{s}^{(2)}\right)_{z z z} & =N_{s} \alpha_{z^{\prime} z^{\prime} z^{\prime}}^{(2)}\left\langle\cos ^{3} \theta\right) \\
\left(\chi_{s}^{(2)}\right)_{z x x} & =\left(\chi_{s}^{(2)}\right)_{z y y}=(1 / 2) N_{s} \alpha_{z^{\prime} z^{\prime} z^{\prime}}^{(2)}\left\langle\sin ^{2} \theta \cos \theta\right\rangle
\end{aligned}
$$

where $N_{\mathrm{s}}$ is the surface density of the molecules and the angular brackets refer to an orientational average. From equation (A-4), it is seen that the measurements of $\left(\chi_{s}^{(2)}\right)_{z z z}$ and $\left(\chi_{s}^{(2)}\right)_{z x x}$ together with the assumption of a $\delta$-function for the orientational distribution allow us to deduce both $\alpha_{z^{\prime} z^{\prime} z^{\prime}}^{(2)}$ and $\theta$. 


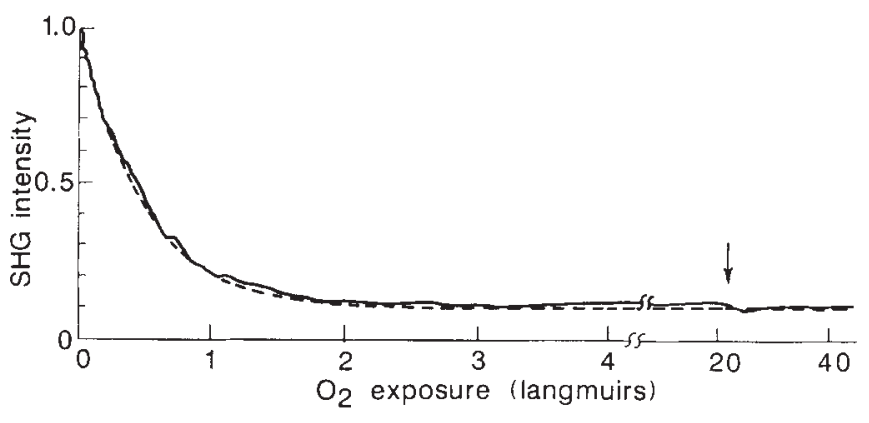

Fig. 2 SHG from a crystalline rhodium surface during $\mathrm{O}_{2}$ exposure ( 1 langmuir unit $=10^{-6}$ torr second). The crystalline surface is specified by the Miller indices $(1,1,1)$, that is, a plane intersecting the three orthogonal crystal axes at points equidistant from the origin. A full monolayer of oxygen on $\mathrm{Rh}(111)$ is characterized by a $(2 \times 2)$ LEED pattern, observed when $\mathrm{O}_{2}$ exposure reaches $\sim 20$ langmuir units, indicated by an arrow. The theoretical curve (dashed line) used to fit the experimental result (solid line) was derived from the simpler Langmuir kinetic model.

modern surface science is the study of metal surfaces ${ }^{1}$. Extensive studies of surface properties and molecule-substrate interactions on well-defined metal surfaces have been carried out in ultrahigh-vacuum (UHV) conditions using conventional surface probes. The SHG technique can be adopted for tasks such as probing the molecular adsorption and desorption, processes involved in all surface problems of practical importance. Figure 2 shows an example of SHG used in UHV conditions to probe molecular adsorption on a metal surface, in this case oxygen on rhodium $^{17}$ The signal from the surface decreases as the surface exposure to oxygen increases. This is qualitatively understood in terms of the fact that the nearly free electrons of a metal often dominate the surface nonlinearity and the adsorption of oxygen tends to localize the free electrons, hence reducing the nonlinearity. Measurement of the surface coverage by oxygen can be calibrated by low-energy electron diffraction (LEED). A full monolayer of oxygen, characterized by a LEED pattern that indicates $(2 \times 2)$ epitaxy, is observed for an oxygen exposure of $\sim 20$ langmuir units $\left(=20 \times 10^{-6}\right.$ torr s). As it is known that the $\mathrm{O}_{2}$ molecule dissociates to atomic oxygen on a rhodium surface the result illustrated in Fig. 2 establishes three important facts: SHG has a sub-monolayer sensitivity; it can monitor the adsorption of atomic species; and it can follow the variation of surface coverage with time. The experimental data in Fig. 2 can be well fitted by a theoretical curve (dashed line) derived from the simple Langmuir kinetic model for adsorption. The 'sticking coefficient' for oxygen on the rhodium surface can be derived from this fit.

Several general properties of the technique are revealed by similar measurements carried out on other adsorbate/metal systems. SHG is sensitive to molecular adsorption at different sites $^{17}$. It correlates well with results from thermal desorption spectroscopy and can be calibrated against the latter in determining surface coverage by adsorbates ${ }^{18}$. More accurate measurements of SHG can, in some cases, reveal the coverage dependence of the sticking coefficients ${ }^{19}$. SHG can probe surface structural changes and phase transitions of adsorbate overlayers ${ }^{20}$. Finally, it can provide information on surface electron resonances $^{21-23}$ and surface structure of metals ${ }^{24}$. In the future, the ultrafast response capability of the technique can be exploited to monitor surface reactions and surface dynamics on metals.

Semiconductor surfaces. The probing of semiconductor surfaces and interfaces is of great relevance because it can provide information about molecular adsorption, surface structure and surface processes. As with metals, molecular adsorption on silicon and germanium can easily be observed with $\mathrm{SHG}^{25,26}$. The dangling bonds on clean silicon and germanium are respon-

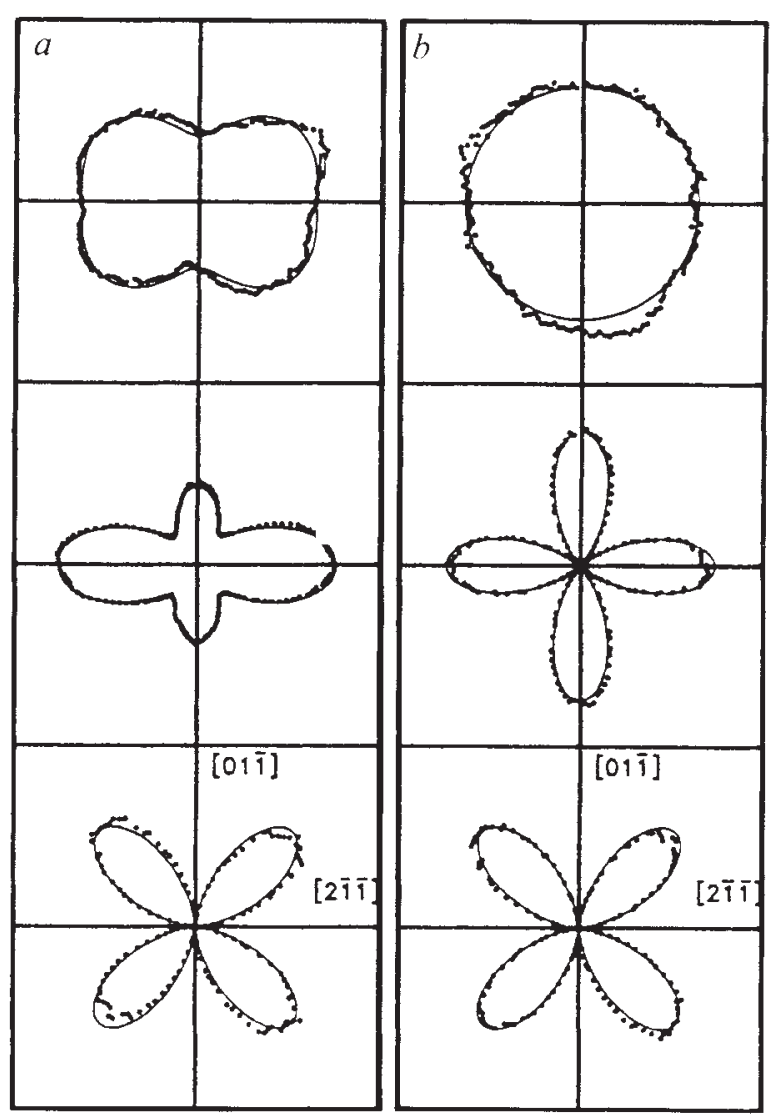

Fig. 3 Polar maps of SHG from $a$, a Si(111) surface characterized by a $(2 \times 1)$ LEED pattern, and $b$, a Si(111) surface characterized by a $(7 \times 7)$ LEED pattern; the polar angle denotes the direction of polarization of the normally incident pump beam. [2 $\overline{1} \overline{1}]$ and [011] specify two orthogonal directions in the crystal. The top panel displays the total second-harmonic signal; the middle and lower panels show, respectively, the signal polarized along [2īi] and $[01 \overline{1}]$. Dashed lines are experimental data and solid lines represent theoretical calculations.

sible for the rather large surface nonlinearities, because of the asymmetrical electron distribution at these bonds. Chemisorbed species that bind to the dangling bonds will reduce the asymmetry and hence the surface SHG. With 1.06- $\mu$ m laser excitation, SHG from a clean silicon surface is completely dominated by the dangling bonds, so that the signal is nearly extinguished by adsorption of a full monolayer ${ }^{24}$.

Surface structure governs the surface properties of a semiconductor. SHG can be used to probe the structural symmetry of a semiconductor surface layer and to monitor phase transitions in situ ${ }^{26}$. This is reflected in the variation of the SHG signal as the sample or the beam polarization rotates about the surface normal $^{27}$. For example, a freshly cleaved $\mathrm{Si}(111)$ surface has structure characterized by a $(2 \times 1)$ LEED pattern, whereas the annealed surface has a structure characterized by a $(7 \times 7)$ LEED pattern. These two surface structures yield very different results of SHG as a function of rotation of the incoming beam polarization (Fig. 3) . $^{26}$. On annealing, the freshly cleaved surface transforms from a $(2 \times 1)$ to a $(7 \times 7)$ structure; the process can be monitored in situ by the variation of SHG with time.

Other surface processes on semiconductors, such as surface melting ${ }^{28-31}$, surface disordering ${ }^{32}$ amorphous-crystalline transformation $^{32}$, formation of metal overlayers ${ }^{32,33}$ and change of surface electronic properties by adsorption $(\mathrm{H}$. W. K. Tom and G. D. Aumiller, personal communication) can also be probed by SHG. Future applications should include monitoring of epitaxial growth, chemical vapour deposition, plasma deposition and etching, and growth of metal-semiconductor junctions. 
The SHG technique as described so far does not work on semiconductors possessing no inversion symmetry, because the bulk contribution to the signal is overwhelming. It has, however, been demonstrated recently that in such cases, the bulk contribution can effectively be suppressed by polarization discrimination, thus regaining the surface sensitivity ${ }^{34}$.

Liquid/solid interfaces. Although liquid/solid interfaces are generally considered as the most important area of surface science, the field has been little explored because of the lack of suitable tools. Surface SHG as a coherent optical technique seems ideal for providing information such as the free energy of adsorption and orientation of adsorbed molecules at interfaces. An example of the first is presented in Fig. 4, where the surface coverage of $p$-nitrobenzoic acid (PNBA) on fused quartz in an ethanol solution, as deduced from $\mathrm{SHG}$, is plotted against the concentration of PNBA in the solution ${ }^{35}$. Such a plot constitutes an adsorption isotherm, from which the free energy of adsorption for the system can be derived. PNBA molecules in the solution contribute little to the signal, confirming the surface-specific nature of the technique.

The orientation of molecular adsorbates at an interface reveals important information about their physical and chemical properties, and about the interaction between the adsorbates and the bonding medium. The molecular orientation at an interface, together with the nonlinear polarizability of the molecules, can sometimes be obtained from the surface SHG measurements (see panel). For the example shown in Fig. 4, the measurements yielded the elements of the surface susceptibility tensor $\left(\chi_{\mathrm{s}}^{(2)}\right)_{z z z}=3.2 \times 10^{-16}$ e.s.u. and $\left(\chi_{\mathrm{s}}^{(2)}\right)_{z x x}=$ $\left(\chi_{\mathrm{s}}^{(2)}\right)_{x y x}=9.0 \times 10^{-17}$ e.s.u. for $0.53-\mu \mathrm{m}$ laser excitation. With a surface density of molecules $N_{\mathrm{s}}=2 \times 10^{14} \mathrm{~cm}^{-2}$, these results yield $\alpha_{z^{\prime} z^{\prime} z^{\prime}}^{(2)}=4 \times 10^{-30}$ e.s.u. and a tilt angle $\theta \approx 40^{\circ}$ (see panel and Fig. $1 b$ ). A similar experiment on PNBA at the air/fused quartz interface gave $\theta \approx 70^{\circ}$. The smaller value for $\theta$ for the liquid/solid interface presumably results from dielectric screening effect of the molecule-substrate interaction by the liquid.

Electrochemistry. Although possessing a long history, electrochemistry is poorly understood at the microscopic level. The enhancement of the SHG signal on roughened metal surfaces ${ }^{15.36}$ should make it easy to detect the appearance of a sub-monolayer of adsorbates on a metal electrode in an electrolyte and thus to monitor molecular adsorption and desorption in such systems.

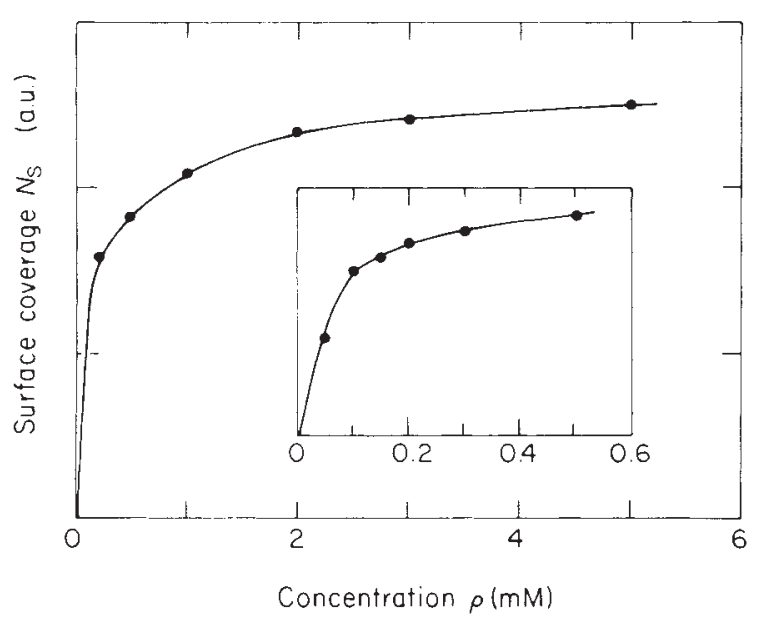

Fig. 4 Adsorption isotherm (surface coverage $N_{\mathrm{s}}$ plotted in arbitrary units as a function of volume concentration $\rho$ ) of $p$ nitrobenzoic acid (PNBA) adsorbed from ethanol solution by fused silica. The surface coverage of PNBA can be deduced from SHG under $s$-polarized excitation at $532 \mathrm{~nm}$, as the SHG signal is proportional to the square of the surface coverage. The inset describes the data points at low concentrations. Coverage is in arbitrary units (a.u.).
Figure 5 shows a representative case, in which the surface SHG from a silver electrode in a $0.1 \mathrm{KCl}$ solution is found to respond to the appearance and disappearance of $\mathrm{AgCl}$ on the electrode $^{37}$. In the oxidation-reduction cycle, the signal increases rapidly when the first monolayer of $\mathrm{AgCl}$ is deposited (as deduced from the amount of charge transfer through the electrode) and decreases rapidly when the last monolayer of $\mathrm{AgCl}$ is reduced. In between these two processes, hundreds of $\mathrm{AgCl}$ layers are deposited on and removed from the electrode, but the signal varies only slightly. This again demonstrates of the surface-specific nature of the technique.

Although SHG should have enough sensitivity to follow the adsorption of the first molecular monolayer on any electrode in any electrolytic process. Several different molecular species may coexist on the electrode and SHG is not selective enough to distinguish one from the others. Interpretation of the results then has to rely on identification of the species obtained by other methods.

In several recent experiments, the effective surface charge density resulting from ions adsorbed on an electrode has been deduced using the dependence of surface SHG on the bias voltage $^{39-41}$. The dynamics of the adsorption and desorption processes can be studied by time-resolved SHG measurements ${ }^{42}$. The technique has also been used to study electrodeposition of metal overlayers on an electrode in an electrolytic solution ${ }^{42-44}$, and to monitor the changes of surface symmetry ${ }^{45}$ and surface properties of a crystalline electrode that result from a change in the bias voltage ${ }^{46,47}$ or from the deposition of metal overlayers ${ }^{44,48}$

Gas/liquid interfaces. Studies of molecular monolayers at gas/liquid interfaces can provide an insight into the understanding of the surface structure of liquids, surfactants, microemulsions and membranes, and the nature of wetting and twodimensional phase transitions. Better control of Langmuir-type
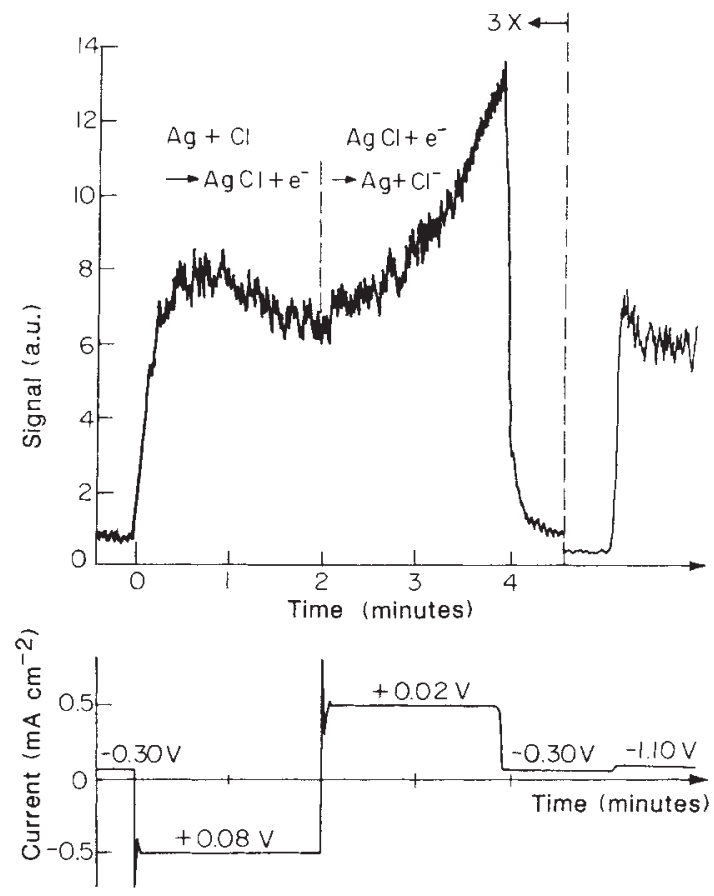

Fig. 5 SHG signal (top) and electrolytic current (bottom) from a silver electrode in a $0.5 \mathrm{M} \mathrm{KCl}$ solution as functions of time during an electrolytic cycle. The voltages listed on the lower curve indicate the potential of the silver electrode with respect to a saturated calomel electrode. The SHG signal is particularly sensitive to the initial deposition and final reduction of $\mathrm{AgCl}$ layers on the electrode. To the left of the vertical dashed line, the SHG signal is exaggerated by a factor of three. 
molecular monolayers on liquid surfaces could yield highquality Langmuir-Blodgett films, important in molecular-electronics applications. SHG is well established as an effective probe for gas/liquid interfaces and only a few demonstrations are described here.

SHG can be used to study phase transition in a monolayer of amphiphilic molecules on water. Measurement of the twodimensional 'surface pressure' $(\pi)$ as a function of surface area $(A)$ of such a system often exhibits not only a two-dimensional gas-liquid phase transition but also, in some cases, a liquid expanded (LE)-liquid condensed (LC) phase transition. It was thought that the latter may be correlated with change in molecular orientation. As SHG can be used to determine molecular orientation, it is an ideal tool for this investigation. Such an experiment on pentadecanoic acid did indeed show a sudden orientational variation associated with the LE-LC transition $^{49}$.

The polymerization of a monolayer on water can also be monitored in situ with SHG. This is interesting because polymerization of a two-dimensional system is expected to be very different from the three-dimensional case. Moreover, the surface density of monomers can easily be varied, and the orientation of the monomers on water may be controlled by molecular synthesis. In such an experiment ${ }^{50}$, a monolayer of octadecyl methacrylate monomers was spread on water and polymerized by irradiation of ultraviolet light. The surface SHG signal decreased accordingly and the time dependent results provided a check for existing theoretical models.

The rate constant of a surface reaction can be very different to that in the bulk and can be monitored by SHG. This has been clearly demonstrated in a recent experiment on a solution of $p$-nitrophenyl in water at various $\mathrm{pH}$ values ${ }^{51}$. Undoubtedly, SHG could also be used to probe liquid/liquid and solid/solid interfaces, but so far there are few examples. Orientations of amphiphilic molecules at different liquid/liquid interfaces, useful for the understanding of micellar or membrane structure, have been measured ${ }^{52}$.

Biological systems. SHG should soon find fruitful applications in biology. For example, molecules adsorbed on membranes tend to have a polar ordering, and should therefore yield a strong signal. SHG has been used to study adsorption of a monolayer of retinal chromophores on water, and on retinal chromophores first embedded in the purple membrane of Halobacterium halobium and then spread on water (J. Huang, A. Lewis, Th. Rasing, T. Stehlin and Y. R. Shen, unpublished results). The orientation, nonlinear polarizability and optical spectrum of the chromophores were obtained, and the change in the dipole moment of the molecules upon optical excitation could be deduced. Microscope images of biological tissues can also be obtained with SHG, providing structural information on the scale and form of molecular ordering ${ }^{53}$. In this case, however, the signal was generated mostly from the bulk of the tissue.

Surface monolayer microscopy. SHG can be used to probe the morphology of a surface monolayer. This was demonstrated by the observation of the SHG image of a $\sim 8-\mu \mathrm{m}$ laser-ablated hole in a Rhodamine $6 \mathrm{G}$ dye monolayer on fused quartz ${ }^{16}$. By using a focused laser beam, the spatial resolution is limited in principle only by the optical wavelength.

\section{Sum-frequency generation.}

With the help of a tunable laser, SHG can be used for surface spectroscopy ${ }^{54}$. The underlying principle is straightforward: when either of the frequencies $\omega$ or $2 \omega$ is close to that of some molecular excitation, the nonlinear susceptibility $\chi^{(2)}$ should exhibit a resonant enhancement. Spectroscopic study is, however, limited to electronic transitions in the visible, because a high-gain photodetector, such as a photomultiplier, is needed to achieve monolayer sensitivity. To study adsorbed molecules selectively, infrared vibrational spectra are more useful because

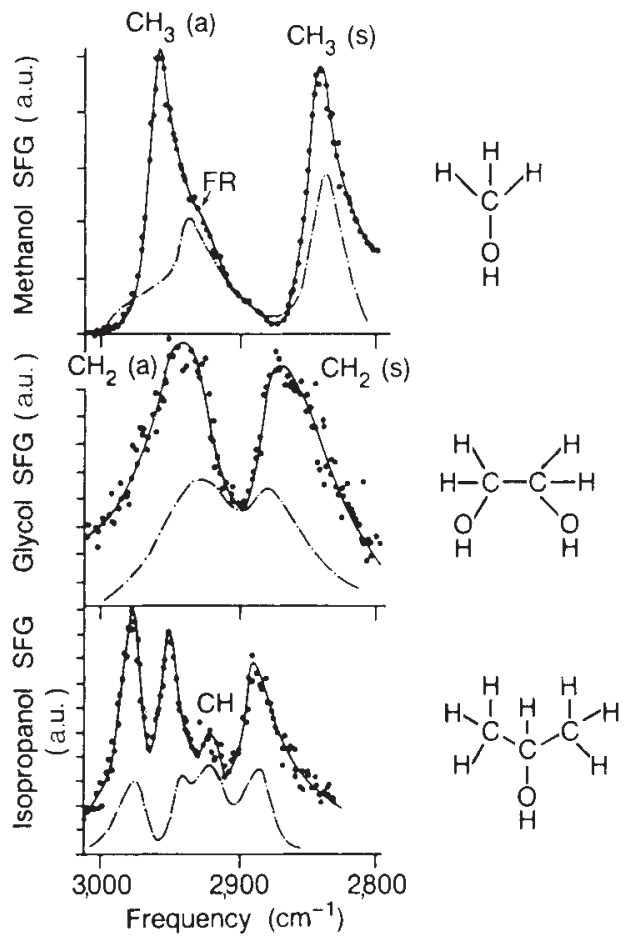

Fig. 6 Sum-frequency generation as a function of infrared input frequency for three adsorbed species, $\mathrm{CH}_{3} \mathrm{OH}, \mathrm{C}_{2} \mathrm{H}_{4}(\mathrm{OH})_{2}$ and $\mathrm{C}_{3} \mathrm{H}_{7} \mathrm{OH}$, on fused silica. The spectra were obtained with a visible input at $0.532 \mu \mathrm{m}$ and a tunable input around $3 \mu \mathrm{m}$. The peaks correspond to the various hydrocarbon $(\mathrm{C}-\mathrm{H})$ stretch modes, symmetric(s) and asymmetric(a); FR denotes a structure resulting from Fermi resonance between $\mathrm{CH}_{3}(\mathrm{~s})$ and the overtone of the $\mathrm{C}-\mathrm{H}$ bending modes. For comparison the Raman spectra of the species in the liquid phase (dashed-dot curves) are also shown.

they constitute 'fingerprints' for identifying molecules but appropriate photomultiplier is available in the infrared. An alternative method, known as sum-frequency generation (SFG), has recently been developed to overcome this problem ${ }^{55-57}$.

Any effective nonlinear optical method for surface vibrational spectroscopy should satisfy three criteria: it should be a secondorder process (that is, responsive to anisotropy) so that it is surface-specific; input must have a tunable infrared component to excite vibrational transitions; and the output should be in the near-infrared or visible, where it can be detected by a photomultiplier. Infrared-visible SFG is an excellent candidate and the technique is a simple extension of SHG. The incoming field $\mathbf{E}$ is of two frequencies, $\omega_{1}$ and $\omega_{2}$; the output is then no longer at $2 \omega$ but at $\omega_{1}+\omega_{2}$, or originating from the surface nonlinear polarization $\mathbf{P}_{\mathrm{s}}^{(2)}\left(\omega_{1}+\omega_{2}\right)$ induced by the incoming fields. If $\omega_{1}$ is in the infrared and $\omega_{2}$ in the visible, all three of the above criteria are satisfied by SFG. Thus, SFG shares the surface specificity of SHG and in addition has capable of selective detection of molecules through their characteristic vibrational transitions.

Surface vibrational spectroscopy by SFG has been applied to the study of molecular adsorbates at various interfaces. Figure 6 shows the SFG spectra of $\mathrm{C}-\mathrm{H}$ bond stretching vibrations of three different molecular species adsorbed on fused quartz ${ }^{58}$. The different stretching modes are clearly resolved and the species can be distinguished by their spectra. The example depicted in Fig. $7 a$ confirms the surface sensitivity of the method. Here the SFG spectra of $\mathrm{C}-\mathrm{H}$ stretches are plotted for three liquid/solid interfacial systems: hexadecane/silica, hexdecane/OTS/silica and $\mathrm{CCl}_{4} / \mathrm{OTS} /$ silica, where OTS denotes a full monolayer of octadecyltrichlorosilane adsorbed on silica ${ }^{59}$. Because surface SFG, like SHG, receives little contribution from 


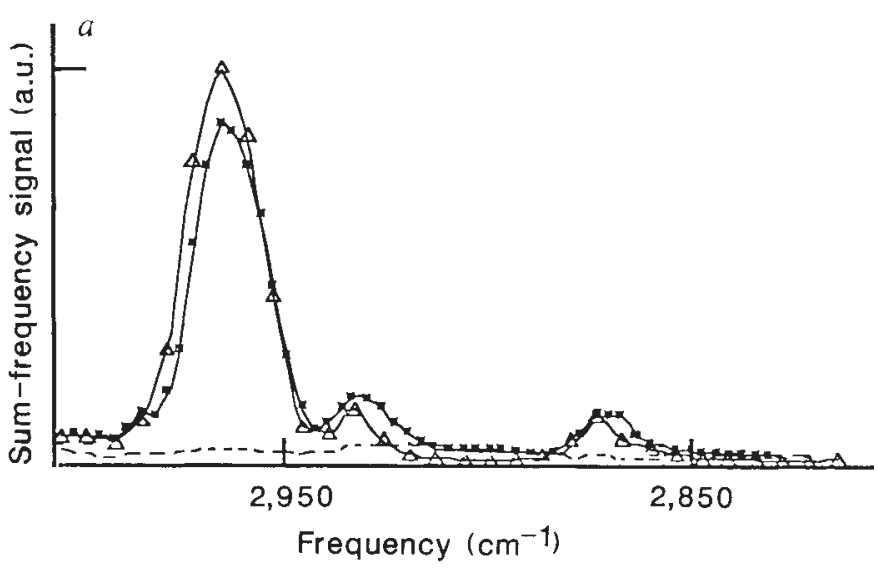

Fig. $7 a$, SFG spectra of different interfaces obtained with a $\hat{p}$-polarized visible input at $0.532 \mu \mathrm{m}$ and a $\hat{p}$-polarized tunable input around $3 \mu \mathrm{m}$. Dashes: hexadecane/silica; solid squares: hexadecane/OTS/silica; triangles: $\mathrm{CCl}_{4} /$ OTS/silica; OTS denotes a monolayer of octadecyltrichlorosilane adsorbed on silica. The solid lines are guides for the eye. OTS, when present, dominates the spectra. $b$, Sum-frequency spectra at the air/OTS/silica interface for various polarization combinations of the visible $(0.532 \mu \mathrm{m})$ and infrared input beams. Left to right, visible: $\hat{p}$-polarized, infrared: $\hat{p}$-polarized; visible: $\hat{p}$, infrared: $\hat{s}$; visible: $\hat{s}$, infrared: $\hat{p}$. The peaks in the spectra can be assigned to $\mathrm{C}-\mathrm{H}$ stretch modes of the terminal methyl group of OTS. The peak at $2,878 \mathrm{~cm}^{-1}$ originates from a $\mathrm{CH}_{3} \mathrm{~s}$-stretch, that at $2,964 \mathrm{~cm}^{-1}$ from a $\mathrm{CH}_{3}$ a-stretch, and that at $2,942 \mathrm{~cm}^{-1}$ from the Fermi resonance between $\mathrm{CH}_{3}(\mathrm{~s})$ and the overtone of the $\mathrm{C}-\mathrm{H}$ bending mode. Peaks for the $\mathrm{CH}_{2}$ stretches on the alkane chain of OTS are hardly visible for reasons of symmetry.

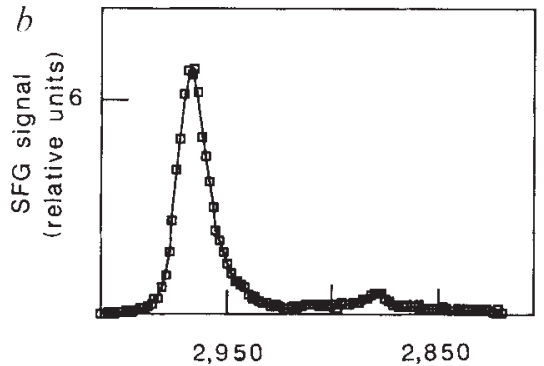

2,950
2,850

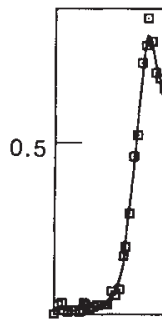

2,950 Frequency $\left(\mathrm{cm}^{-1}\right)$

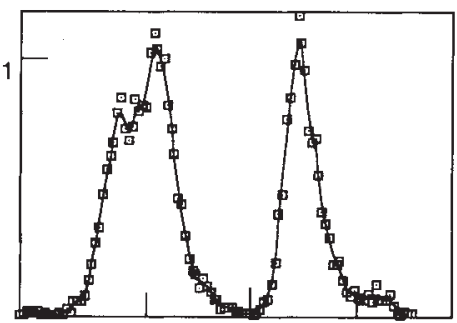

2,950

2,850 the isotropic bulk, the spectrum for the first system shows no visible peaks, even though liquid hexadecane should have a strong infrared absorption band in this spectral region arising from the large number of $\mathrm{C}-\mathrm{H}$ stretches in these molecules. Comparison of the two spectra for $\mathrm{CCl}_{4} / \mathrm{OTS} /$ silica and hexadecane/OTS/silica also demonstrates the surface specificity: the two spectra are nearly identical, both exhibiting the stretch modes only of the terminal methyl group of OTS.

The polarization dependence of SFG spectra also yields useful information. Figure $7 b$ shows the spectra of the air/OTS/silica interface for three different polarization combinations ${ }^{59}$. The peak heights are clearly different in each case. All the peaks in the spectra are again associated with the $\mathrm{C}-\mathrm{H}$ stretches of the terminal methyl group of OTS, and the polarization dependence results from the orientation of this group. A quantitative analysis can be performed to calculate this orientation. In Fig. $7 b$, the data yield an angle of $\sim 45^{\circ}$ between the $\mathrm{CH}_{3}$ symmetric axis and the surface normal, indicating that, as expected, the alkyl chain of OTS is nearly parallel to the surface normal.

SFG surface vibrational spectroscopy has also been applied to metal and semiconductor surfaces ${ }^{60,61}$. The use of subpicosecond pump pulses further permits the study of surface dynamics of selected molecular species. The fast response of SFG may even provide a means for following selective surface reactions and for probing intermediate species in the reactions. At present, the main difficulty of this technique is the lack of tunable sources in the mid- and far-infrared range but this problem should be overcome when infrared free-electron lasers become generally available.

In summary, SHG is an extremely versatile and valuable tool for surface studies that can be applied to all interfaces accessible to light and has many unique advantages over conventional surface probes. In particular, its sub-picosecond time resolution offers possibilities for research on ultrafast surface dynamics and reactions. The lack of molecular selectivity is overcome by using SFG for surface vibrational spectroscopy. These nonlinear optical techniques should soon be widely adopted for surface and interface studies in many disciplines.

This work was supported by the Direction, Office of Energy Research, Office of Basic Energy Sciences, Materials Sciences Division of the US Department of Energy.

1. Somorjai, G. Chemistry in Two Dimensions: Surfaces (Cornell University Press, New York 1981).

2. Aspnes. D. E. \& Studna A. A. Phys Rev. Lett 54, 1956-1959 (1985).

3. Hail, R. B. \& Ellis, A. B. (eds) New laser and Optical lnvestigation of Chemistry and Structure at Interfaces (Deerfield Beach, Florida, 1986).

4. Bloembergen, N., Chang, R. K., Jha, S. S. \& Lee, C. H. Phys. Rev. 174, 813-822 (1968)

5. Wang, C. C. Phys. Rev. 178, 1457-1461 (1969)

6. Guyot-Sionnest, P., Chen, W. \& Shen, Y. R. Phys. Rev. B 33, 8254-8263 (1986)

7. Guyot-Sionnest, P. Shen, Y. R. Phys. Rev. B 35, 4420-4426 (1987)

8. Bloembergen, N. \& Pershan, P. S. Phys. Rev. 128, 606-622 (1962)

9. Shen, Y. R The Principles of Nonlinear Optics Ch. 25 (Wiley, New York, 1984)

9. Shen, Y. R. The Principles of Nonlinear Oprics Ch. 5 (Wil. Commun. 9, 132-134 (1973).

11. Fleischmann, M., Hendra, P. J. \& McQuillian, A. J. Chem. Phys. Lett. 26, 163-166 (1974).

12. Jeanmaire, D. \& van Dyne, R. P. J. electroanalyt. Chem. 84, 1-20 (1977).

13. Moskovits, M. J. chem. Phys. 69, 4159-4161 (1978).

14. Gersten, J. 1. \& Nitzan, A. J. chem. Phys. 73, 3023-3037 (1980).

15. Chen, C. K., de Castro, A. R. B. \& Shen, Y. R. Phys. Rev. Lett. 46, 145-148 (1983).

16. Boyd, G. T., Shen, Y. R. \& Hansch, T. W. Opt. Lett. 11, 97-99 (1986).

17. Tom, H. W. K et al Phys, Rev, Lett 52, 348-351 (1984).

18. Zhu, X. D., Shen, Y. R. \& Carr, R. Surf. Sci. 163, 114-120 (1985).

19. Grubb, S. G., DeSantolo, A. M. \& Hall, R. B. J. phys. Chem, 92, 1419-1425 (1988)

20. Heskett, D., Song, K. J., Burns, A., Plummer, E. W. \& Dai, H. L. J. chem. Phys. 85, 7490-7491 (1986).

21. Tom, H. W. K. et al Surf. Sci. 172, 466-476 (1986).

22. Hener, W., Schröder \& Zacharias, H. Chem. Phys, Lett. 135, 299-302 (1987).

23. Giesen, K. et al. Physica Scripta 35, 578-581 (1987).

24. Tom, H. W. K. \& Aumiller, G. D. Phys. Rev. B 33, 8818-8821 (1986)

24. Tom, H. W. K. \& Aumiler, G. D. Phys. Rev. B 33, 8818-8821 (1986).
25. Tom, H. W. K., Zhu, X. D., Shen, Y. R. \& Somorjai, G. A. Surf. Sci, 167, 167-176 (1986).

25. Tom, H. W. K., Zhu, X. D., Shen, Y. R. \& Somorjai, G. A. Surf. Sci, 167, 167-176 (198
26. Heinz, T. F., Loy, M. M. T. \& Thompson, W. A. Phys. Rev. Lett. 54, 63-66 (1985).

27. Tom, H. W. K., Heinz, T. F. \& Shen, Y. R., Phys. Rev. Lett. 51, 1983-1986 (1983)

28. Shank, C. V., Yen, R. \& Hirlimann, C. Phys. Rev. lett. 51, 900-902 (1983).

29. Akhamanov, S. A. et al. Opt. Commun. 47, 202-204 (1983).

30. Malvezzi, A. M., Liu, J. M. \& Bloembergen, N. Appl. Phys. Lett. 45, 1019-1021 (1984).

31. Tom, H. W. K., Aumiller, G. D. \& Brito-Cruz, C. H. Phys, Rev. Lett 60, 1438-1444 (1988)

32. Heinz, T. F., Loy, M. M. T. \& Thompson, W. A., J. Vac. Sci. Technol. B3, 1467-1470 (1985)

33. lyer, S. S., Heinz, T. F. \& Loy, M. M. T., J. Vac. Sci. Technol. B5, 709-710 (1987).

34. Stehlin, T., Feller, M., Guyot-Sionnest, P. \& Shen, Y. R. Opt. Lett. 13, 389-391 (1988).

35. Heinz, T. F., Tom, H. W. K. \& Shen, Y. R. Phys. Rev. A 28, 1883-1885 (1983).

36. Boyd, G. T., Rasing, Th., Leite, J. R. R. \& Shen, Y. R. Phys. Rev. B 30, 519-526 (1984).

37. Chen, C. K., Heinz, T. F., Ricard, D. \& Shen, Y. R. Phys. Rev. Lett. 46, 1010-1012 (1981)

38. Murphy, D. V., von Raben, K. U., Chen, T. T., Owen, J. F. \& Chang, R. K. Surf. Sci, 124 529-546 (1983).

39. Corn, R. M., Romagnoli, M., Levenson, M. D. \& Philpott, M. R., Chem. Phys. Lett. 106, $30-35(1984)$. 
40. Richmond, G. L. Chem. Phys. Lett. 110, 571-575 (1984).

41. Richmond, G. L., Rojhantalab, H. M., Robinson, J. M. \& Shannon, V. L. J. Opt. Soc. Am. B4, 228-236 (1987).

42. Corn, R. M., Romagnoli, M., Levenson, M. D. \& Philpott, M. R. J. chem. Phys. 81, 4127-4132 (1984).

43. Furtak, T. E., Meragliotta, J. \& Korenowski, G. M. Phys. Rev. B 35, 2596-2572 (1987). 44. Richmond, G. L., Koos, D. A., Robinson, J. M. \& Shannon, V. L., Bull. Am. phys. Soc. 33, 1648 (1988).

45. Shannon, V. L., Koos, D. A. \& Richmond, G. L. J. chem. Phys. 87, 1440-1441 (1987); Appl Opt. 26, 3579-3583 (1987).

46. Shannon, V. L., Koos, D. A. \& Richmond, G. L. J. phys. Chem. 91, 5548-5555 (1987).

47. Shannon, V. L., Koos, D. A., Robinson, J. M. \& Richmond, G. L. Chem. Phys. Leti. 142, 323-328 (1987)

48. Miragliotta, J. \& Furtak, T. E. Phys, Rev. B 37, 1028-1030 (1988).

49. Rasing. Th., Kim, M. W., Shen, Y. R. \& Grubb, S. Phvs. Rev. Lett. 55, 2903-2906 (1985)
50. Berkovic, G., Rasing, Th. \& Shen, Y. R. J. chem. Phys, 85, 7374-7376 (1986).

51. Bhattacharyya, K Sitzmann, E V \& Eisenthal, K. B. J chem. Phys. 87, 1442-1443 (1987).

52. Grubb, S. G., Kim, M. W., Rasing, Th. \& Shen, Y. R. Langmuir 4, 452 454 (1988).

53. Freund, I. \& Deutsch, M. Opt. Lett. 11, 94-96 (1986).

54. Heinz, T. F., Chen, C. K., Ricard, D. \& Shen, Y. R. Phys. Rev. Lett. 48, 478-48I (1983).

55. Zhu, X. D., Suhr, H. \& Shen, Y. R. Phys. Rev. B 35, 3047-3050 (1987).

56. Hunt, J. H., Guyot-Sionnest, P. \& Shen, Y. R. Chem. Phys. Letl. 133, 189-192 (1987).

57. Guyot-Sionnest, P. Hunt, J. H. \& Shen, Y. R. Phys. Rev. Lett 59, 1597-1600 (1987).

88. Hunt, J. H., Guyot-Sionnest, P. \& Shen, Y. R. in Laser Spectroscopy VIII (eds Persson, W. \& Svanberg, S.) 253-266 (Springer, Berlin, 1987).

59. Guyot-Sionnest, P., Superfine, R. \& Hunt, J. H. Chem. Phys. Lett. 144, 1-5 (1988)

60. Harris, A. L., Chidsey, C. E. D., Levinos, N. J. \& Loiacono, D. N. Chem. Phys. Lett. 141, 350-356 (1987)

61. Superfine, R., Guyot-Sionnest, P., Hunt, J. H., Kao, C. T. \& Shen, Y. R. Surf. Sci. 200, L445-L450 ( 1988 .

ARTICLES

\title{
Designing CD4 immunoadhesins for AIDS therapy
}

\author{
Daniel J. Capon, Steven M. Chamow ${ }^{*}$, Joyce Mordenti ${ }^{\dagger}$, Scot A. Marsters, \\ Timothy Gregory*, Hiroaki Mitsuya ${ }^{\dagger}$, Randal A. Byrn ${ }^{\S}$, Catherine Lucas", \\ Florian M. Wurm", Jerome E. Groopman ${ }^{\S}$, Samuel Broder ${ }^{\dagger} \&$ Douglas H. Smith
}

Departments of Molecular Biology, * Recovery Process Research and Development, $\uparrow$ Pharmacological Sciences, $\|$ Medicinal and Analytical Chemistry, $\uparrow$ Cell Culture Research and Development, Genentech, Inc., 460 Point San Bruno Boulevard, South San Francisco, California, 94080, USA

$\ddagger$ The Clinical Oncology Program, National Cancer Institute, National Institutes of Health, Bethesda, Maryland, 20892, USA

$\S$ Division of Hematology-Oncology, Harvard Medical School, New England Deaconess Hospital, Boston, Massachusetts, 02215, USA

A newly-constructed antibody-like molecule containing the gp120-binding domain of the receptor for human immunodeficiency virus blocks HIV-1 infection of $T$ cells and monocytes. Its long plasma half-life, other antibody-like properties, and potential to block all HIV isolates, make it a good candidate for therapeutic use.

DESPITE the exquisite ability of the immune system to distinguish between self and non-self, and to put forth an impressive diversity in its antigen-recognizing repertoire, it can still be outflanked by a rapidly changing pathogen. Human immunodeficiency virus type 1 (HIV-1) is an example of such a pathogen, and, as a result, its consequences are devastating. Every individual infected with the virus is expected to develop a serious or life-threatening illness ${ }^{1}$; no protective state has been shown to be generated in natural infections. It has not yet been possible to generate a protective response by immunizing chimpanzees with gp120, the HIV-1 envelope glycoprotein ${ }^{2,3}$, or to confer passive immunity to chimpanzees using human $\mathrm{IgG}^{4}$. Even neutralizing antibodies made in experimental animals can block the infectivity of only a few HIV-1 isolates ${ }^{3,5}$. Thus, the prospects for eliciting protective immunity against HIV-1, or for using antibodies as therapeutic agents to control HIV-1 disease are bleak. Anti-retroviral chemotherapy using dideoxynucleosides such as AZT does help some patients, but the toxicity is such that new strategies are needed ${ }^{6}$.

We have therefore attempted to block HIV-1 infectivity with soluble derivatives of CD4, the receptor for HIV-1, with the rationale that the CD4-binding domain of gp120 is the only part of gp120 that the virus cannot afford to change ${ }^{7}$. CD4 is a cell-surface glycoprotein found mostly on a subset of mature peripheral $\mathrm{T}$ cells that recognize antigens presented by class II MHC molecules ${ }^{8,9}$. Antibodies to CD4 block HIV-1 infection of $\mathrm{T}$ cells ${ }^{10,11}$ and human cells not susceptible to HIV-1 infection become so after transfection with a CD4 cDNA ${ }^{12}$. Gp120 binds CD4 with high affinity $\left(K_{\mathrm{D}} \sim 10^{-9} \mathrm{M}\right)$, suggesting that it is this interaction which is crucial to the entry of virus into cells ${ }^{7,13}$. Indeed, $\mathrm{we}^{7}$ and others ${ }^{14-18}$ have shown that soluble rCD4, lacking the transmembrane and cytoplasmic sequences of CD4, can block HIV-1 infectivity, syncytium formation, and cell killing by gp120 (ref. 19). rCD4 blocks the infectivity of diverse HIV-1 isolates (R.B., J.G., H.M. and S.B., unpublished results), and in theory should block all. At best, however, soluble rCD4 offers only a passive defence against the virus.

Active immunity requires a molecule such as an antibody, which can specifically recognize a foreign antigen or pathogen and mobilize a defence mechanism. Antibodies comprise two functionally independent parts, a rather variable domain (Fab), which binds antigen, and an essentially constant domain ( $\mathrm{Fc}$ ), providing the link to effector functions such as complement or phagocytic cells. It is almost certainly the lack of an antigenbinding domain which can neutralize all varieties of virus that hampers the development of humoural immunity to HIV-1. We reasoned that the characteristics of CD4 would make it ideal as the binding site of an antibody against HIV-1. Such an antibody would bind and block all HIV-1 isolates, and no mutation the virus could make, without losing its capacity to infect $\mathrm{CD}^{+}$ cells specifically, would evade it. We therefore set out to construct such an antibody by fusing CD4 sequences to antibody domains.

We had two major aims for our hybrid molecules; first, as pharmacokinetic studies in several species predict that the halflife of soluble CD4 will be short in humans (30-120 min; J.M., unpublished results) we wished to construct a molecule with a longer half-life; second, we wanted to incorporate functions such as Fc receptor binding, protein A binding, complement fixation and placental transfer, all of which reside in the $\mathrm{Fc}$ portion of IgG. The Fc portion of immunoglobulin has a long plasma half-life, like the whole molecule, whereas that of Fab is short, and we therefore expected to be able to fuse our short-lived CD4 molecule to Fc and generate a longer-lived CD4 analogue. Because CD4 is itself part of the immunoglobulin gene superfamily, we expected that it would probably fold in a way that is compatible with the folding of Fc. We have therefore produced a number of CD4-immunoglobulin hybrid molecules, using both the light and the heavy chains of immunoglobulin, and investigated their properties. We have named one 